\title{
Global attractors, extremal stability and periodicity for a delayed population model with survival rate on isolated time scales
}

Jaqueline Godoy Mesquita ${ }^{1}$, Ewa Schmeidel ${ }^{2}$, Urszula Ostaszewska ${ }^{3}$, and Malgorzata Zdanowicz $^{2}$

${ }^{1}$ Universidade de Brasilia

${ }^{2}$ University of Bialystok, Poland

${ }^{3}$ University of Bialystok

October 21, 2020

\begin{abstract}
In this paper, we investigate the existence of global attractors, extreme stability, periodicity and asymptotically periodicity of solutions of the delayed population model with survival rate on isolated time scales given by $\backslash\left[\mathrm{x}^{\wedge}\{\backslash\right.$ Delta $\}(\mathrm{t})=\backslash$ gamma $(\mathrm{t}) \mathrm{x}(\mathrm{t})$ $+\backslash \operatorname{dfrac}\{\mathrm{x}(\mathrm{d}(\mathrm{t}))\}\{\backslash \operatorname{mu}(\mathrm{t})\} \mathrm{e}^{\wedge}\{\mathrm{r}(\mathrm{t}) \backslash \operatorname{mu}(\mathrm{t}) \backslash \operatorname{left}(1-\backslash \operatorname{frac}\{\mathrm{x}(\mathrm{d}(\mathrm{t}))\}\{\backslash \mathrm{mu}(\mathrm{t})\} \backslash$ right $)\}, \backslash \backslash \mathrm{t} \backslash$ in $\backslash$ mathbb $\mathrm{T}$. $\left.\backslash\right]$ We present many examples to illustrate our results, considering different time scales
\end{abstract}

\section{Hosted file}

Schmeidel.pdf available at https://authorea.com/users/369205/articles/488132-globalattractors-extremal-stability-and-periodicity-for-a-delayed-population-model-with-

survival-rate-on-isolated-time-scales 
figures/solu1/solu1-eps-converted-to.pdf 
figures/solu2/solu2-eps-converted-to.pdf 
figures/solu3/solu3-eps-converted-to.pdf 
figures/solu4/solu4-eps-converted-to.pdf 
figures/solut5/solut5-eps-converted-to.pdf 